\section{At the intersection of primary pulmonary myxoid sarcoma and pulmonary angiomatoid fibrous histiocytoma: observations from three new cases}

DOI: $10.1111 /$ his.12354

(C) 2014 John Wiley \& Sons Ltd.

Sir: Twin recent reports identified a novel entity, primary pulmonary myxoid sarcoma (PPMS) ${ }^{1}$ and an endobronchial presentation of angiomatoid fibrous histiocytoma $(\mathrm{AFH}){ }^{2}$ PPMS were described as 20 40-mm diameter endobronchial, lobulated neoplasms occurring in young patients (mean age 45 years), and comprising polygonal to spindled cells within a myxoid stroma evocative of extraskeletal myxoid chondrosarcoma (EMC). ${ }^{1,3}$ In contrast to EMC, seven of nine testable cases harboured EWSR1-CREB1 fusions. Nine of 10 showed remarkable associated inflammation, which was focal to follicular to lymphoplasmacytic. Two of six patients for whom followup was available developed metastases (brain and kidney), supporting classification as a low-grade sarcoma. ${ }^{1}$ One additional patient had no recurrence. ${ }^{4}$ Two cases of endobronchial $\mathrm{AFH}^{2}$ showed a characteristic multinodular growth pattern of bland, ovoid to spindled cells with a peritumoral lymphoplasmacytic cuff, a fibrotic capsule, and strong focal desmin expression (without classic blood-filled 'angiomatoid' spaces). Similarly to reports of eight AFHs occurring in unusual sites, ${ }^{5}$ including the lung, both endobronchial AFHs showed focal myxoid change. ${ }^{2}$ One lesion harboured an EWSR1-CREB1 fusion, and the other harboured an EWSR1-ATF1 fusion. Neither of the patients (males aged 61 and 64 years) has suffered recurrence. $^{2}$

To emphasize the recent recognition of this entity, we report three additional patients with what we termed PPMS, seen in consultation, recognizing overlap with what might be considered pulmonary AFH. Patient 1 was a 66-year-old woman who presented with a 2-year history of intermittent pulmonary obstructive symptoms and pneumonia, at which time her tumour was discovered on a chest computed tomography (CT) scan. Her resected left upper lobe showed a pale $40-\mathrm{mm}$ diameter endobronchial mass, with associated postobstructive consolidation. Microscopically, her endobronchial-based tumour (Figure 1A) infiltrated into peribronchial pulmonary parenchyma. Over $80 \%$ of the lesion showed polygonal to spindled cells with a reticular, myoepithelialtype appearance and mild atypia in a pink-grey myxoid stroma (Figure 1B). A peritumoral fibrous cuff was present, particularly at the interface with alveolar parenchyma (Figure 1C). The lesion was negative for pancytokeratin, p63, S100, and desmin, and showed patchy EMA-positive cells; break-apart fluorescence in-situ hybridization (FISH) was positive for both EWSR 1 (Figure 1D) and CREB1 (negative for ATF1).

Patient 2 was a 28 -year-old man presenting with 4 months of cough with haemoptysis. Imaging showed an $85-\mathrm{mm}$ diameter right lower lobe mass described grossly as obliterating the bronchus. Microscopically, this tumour was a lobular, biphasic lesion, $\sim 40 \%$ of which was composed of myxoid pools (Figure 1E). The remainder showed exuberant fibroinflammatory reaction with confluent plasma cells. Two foci showed 'angiomatoid' blood-filled spaces (Figure 1F). Moderate atypia was present (more than in patient 1) among reticular and clustered cells floating in the distinctive EMC-type stroma (Figure 1G). The lesion was negative for pancytokeratin, p63 and S100, showed focal EMA positivity, but was strongly positive for desmin, visible against the inflammatory background and discontinuous peritumoral fibrous capsule (Figure 1H). FISH was positive for EWSR1 rearrangement, but not for CREB1 or ATF1 rearrangements.

Patient 3 was another 28-year-old man, who presented with a 2-week history of chest pain. A CT scan showed a $60-\mathrm{mm}$ diameter lesion in the right upper lobe of the lung. At low magnification, the tumour showed a destructive growth pattern, with focal necrosis and infiltration from an endobronchial base into adjacent lung parenchyma (Figure 1I). At higher power, the degree of atypia was severe (Figure 1J), and the myxoinflammatory areas $(80 \%$ of the lesion) expanded to undermine the bronchial epithelium (Figure 1K). As in case 1, the lesion was negative for keratins, p63, and S100, and there were rare EMA-positive cells. FISH was negative for rearrangement at EWSR1 (Figure 1L), CREB1, and ATF1.

Taken together, these lesions span a spectrum from PPMS to AFH in terms of morphology, atypia, and molecular findings. The varying proportion of the myxoid component, from $40 \%$ to $80 \%$, is greater than that described for AFH cases in the lung. ${ }^{2,5,6}$ Case 2 showed a degree of plasmacytic inflammation, a fibrotic component, and desmin positivity, none of which were included in previous descriptions of PPMS, ${ }^{1,4}$ with 'angiomatoid' foci that have not yet been reported in endobronchial $\mathrm{AFH}^{2}$ or PPMS cases. ${ }^{1,4}$ All three cases showed at least a focal peritumoral fibrous 

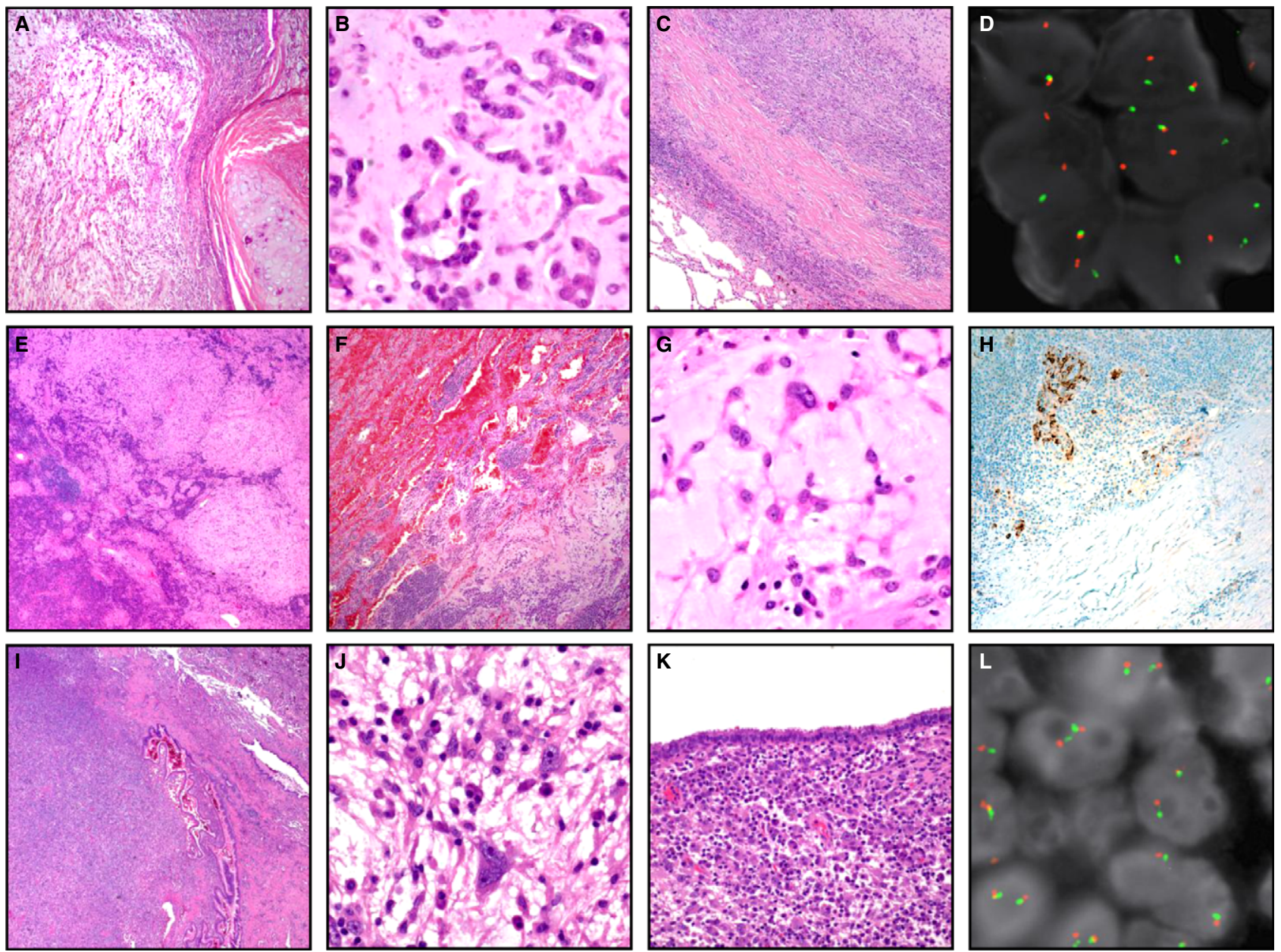

Figure 1. Three cases of primary pulmonary myxoid sarcoma versus angiomatoid fibrous histiocytoma. A-D, Case 1 was an endobronchial lesion with reticular spindle cells in myxoid stroma (A), mild atypia (B), a focal perilesional fibroinflammatory cuff (C), and rearrangement at EWSR1 (D) and CREB1. E-H, Case 2 showed exuberant inflammation (E), angiomatoid areas (F), moderate cellular atypia (G), desmin positivity (H), and rearrangement at EWSR1, but not at CREB1 or ATF1. I-L, Case 3 showed an expansile, high-grade, focally necrotic mass (I) with severe atypia (J), undermining the bronchial epithelium (K); rearrangements at EWSR1 (L), CREB1 or ATF1 were not detected.

cuff, although nodular proliferations of bland ovoid cells characteristic of AFH were only seen focally in case 2. All three lesions were diagnosed as PPMS, to underscore our concern for risk of recurrence (particularly since each tumour was larger than previously reported PPMS or pulmonary AFH cases $\left.^{1,2,4}\right)$, with recognition of the AFH-overlapping features noted especially in case 2 . All three patients are disease free at 18,16 and 4 months post-resection.

We note that EWSR 1 rearrangements were present in only two of our patients. Given prior observation of a subset of PPMS without identifiable rearrangements $^{1}$ and of AFH of unusual sites showing rearrangement at the FUS locus (rather than EWSR 1), ${ }^{5,7,8}$ emerging technologies such as RNA sequencing may be more suitable for comprehensive molecular assessment as a larger number of these cases are assembled and studied. Taken together, the clinical, histopathological and immunophenotypic findings of these cases were sufficiently distinctive to support a diagnosis of PPMS, recognizing the absence of clearly defined diagnostic criteria, particularly with reference to molecular findings. Our experience supports a previous suggestion ${ }^{2}$ that PPMS and AFH of the lung may represent an overlapping morphological and biologic spectrum; multi-institutional efforts to study the morphological, clinical and molecular features of larger cohorts may be helpful in establishing diagnostic criteria and assessing the biological potential of these lesions. 


\section{Author Contributions}

All authors made substantial contributions to the design of the study and the acquisition of data, or analysis and interpretation thereof, assisted in drafting or revising the manuscript, and approved the final version.

\section{Conflict of interest}

The authors declare that there is no conflict of interest.

\section{Steven C Smith ${ }^{1, *}$ Nallasivam Palanisamy ${ }^{1,2, *}$ Bryan L Betz ${ }^{1}$ Scott A Tomlins ${ }^{1,2,3}$ Rohit Mehra ${ }^{1,2}$ Lindsay A Schmidt ${ }^{1}$ David R Lucas ${ }^{1}$ Jeffrey L Myers ${ }^{1}$ Michigan, Ann Arbor, MI, USA}

${ }^{1}$ Department of Pathology, ${ }^{2}$ Michigan Center for Translational Pathology, and ${ }^{3}$ Departments of Urology and Comprehensive Cancer Center, University of

*These authors contributed equally to this study.

1. Thway K, Nicholson AG, Lawson K et al. Primary pulmonary myxoid sarcoma with EWSR1-CREB1 fusion: a new tumor entity. Am. J. Surg. Pathol. 2011; 35; 1722-1732.

2. Thway K, Nicholson AG, Wallace WA, Al-Nafussi A, Pilling J, Fisher C. Endobronchial pulmonary angiomatoid fibrous histiocytoma: two cases with EWSR1-CREB1 and EWSR1-ATF1 fusions. Am. J. Surg. Pathol. 2012; 36; 883-888.

3. Nicholson AG, Baandrup U, Florio R, Sheppard MN, Fisher C. Malignant myxoid endobronchial tumour: a report of two cases with a unique histological pattern. Histopathology 1999; 35; 313-318.

4. Matsukuma S, Hisaoka M, Obara K et al. Primary pulmonary myxoid sarcoma with EWSR1-CREB1 fusion, resembling extraskeletal myxoid chondrosarcoma: case report with a review of literature. Pathol. Int. 2012; 62; 817-822.

5. Chen G, Folpe AL, Colby TV et al. Angiomatoid fibrous histiocytoma: unusual sites and unusual morphology. Mod. Pathol. 2011; 24; 1560-1570.

6. Ren L, Guo SP, Zhou XG, Chan JK. Angiomatoid fibrous histiocytoma: first report of primary pulmonary origin. Am. J. Surg. Pathol. 2009; 33; 1570-1574.

7. Waters BL, Panagopoulos I, Allen EF. Genetic characterization of angiomatoid fibrous histiocytoma identifies fusion of the FUS and ATF-1 genes induced by a chromosomal translocation involving bands $12 \mathrm{q} 13$ and 16p11. Cancer Genet. Cytogenet. 2000; 121; 109-116.

8. Raddaoui E, Donner LR, Panagopoulos I. Fusion of the FUS and ATF1 genes in a large, deep-seated angiomatoid fibrous histiocytoma. Diagn. Mol. Pathol. 2002; 11; 157-162.

\section{A case of oral lichen sclerosus with gingival involvement and Borrelia identification}

\author{
DOI: $10.1111 /$ his.12363
}

(C) 2014 John Wiley \& Sons Ltd.

Sir: Lichen sclerosus (LS) is a chronic mucocutaneous inflammatory disorder that most commonly affects the anogenital skin and occurs more frequently in females than in males. ${ }^{1}$ Clinically, it presents as white, sometimes painful, pruritic papules or plaques. Despite its predilection for the anogenital regions the condition can occur throughout the body, although lesions of the oral mucosa are exceedingly rare and those involving the gingiva are even more so. ${ }^{2,3}$ In the oral mucosa LS is seen typically in middle-aged females, the most common sites being the lower lip, buccal mucosa and upper lip. ${ }^{2}$ An increased risk of genital squamous cell carcinoma (SCC) has been documented in genital LS, thus resulting in an increased need for close follow-up and management of affected patients. ${ }^{1,2}$ Malignancy involving oral cases of LS, however, has not been reported. ${ }^{2}$ First-line treatment of LS typically involves the use of topical corticosteroids; nevertheless, the condition is not cured and remissions do not usually occur. ${ }^{1}$

Histologically, LS is characterized by an atrophic thinned epidermis with blunted rete ridges, hydropic degeneration of the basal layer, and dermal collagen sclerosis with an underlying band of lymphocytic infiltrate. These features tend to overlap in both skin and oral mucosal lesions. ${ }^{2,3}$

The underlying aetiology of LS remains unidentified; however, several possible causes have been suggested, ranging from chronic irritation to an autoimmune component, as well as infectious causes. ${ }^{1}$ Infection with Borrelia burgdorferi, the causative organism of Lyme disease, has been proposed as a possible aetiology, due to a similarity in their histological features and the identification of Borrelia organisms in some cases of LS. ${ }^{4}$ Evidence of this association has remained conflicting over the years, due in part to the overall lack of consistency in identifying Borrelia in many LS cases and in general., ${ }^{1,5}$ Furthermore, LS does not appear to have predominantly infectious characteristics, as there has been only a single case report of LS occurring in sexual partners. ${ }^{5}$ This does not, however, discount the possibility that LS is a reactive process occurring in Borrelia-infected skin or soft tissue. It is interesting to note that the various instances of Borrelia involvement of 